\title{
Self-organized nanostructures in multi-phase epilayers
}

\author{
Dongchoul Kim and Wei $\mathrm{Lu}^{1}$ \\ Department of Mechanical Engineering, University of Michigan, Ann Arbor, MI 48109, USA \\ E-mail: weilu@umich.edu
}

Received 9 September 2003, in final form 6 February 2004

Published 22 March 2004

Online at stacks.iop.org/Nano/15/667 (DOI: 10.1088/0957-4484/15/5/045)

\begin{abstract}
Recent experiments have shown that multi-phase epilayers may self-organize into ordered nanoscale patterns on a solid substrate. In this paper, we present a phase field model for the self-assembly of three-phase epilayers. Nanoscale patterns are developed by two competing actions: coarsening due to phase boundary energy and refining due to substrate-mediated elastic interaction. The continuum phase field approach leads to a set of nonlinear diffusion equations, which couples the two concentration fields in the epilayer and the stress field in the substrate. Numerical simulations reveal remarkably rich dynamics in the self-assembly of multi-phase epilayers. An epilayer may evolve into various patterns, suggesting a significant degree of experimental control in growing nanoscale structures.
\end{abstract}

(Some figures in this article are in colour only in the electronic version)

\section{Introduction}

Advancing technologies demand solid structures of ever decreasing length scales. Self-assembled nanostructures have aroused considerable research interest in recent years, thanks to the advancement of nanoscale microscopy technology, such as STM (scanning tunnelling microscopy) [1]. Experiments have shown that a multi-phase epilayer on a solid substrate may self-organize into various nanoscale patterns. Pohl et al [2] deposited a mixture of $\mathrm{Ag}$ and $\mathrm{S}$ on an $\mathrm{Ru}(0001)$ surface. An ordered triangular lattice of S rich dots formed in the Ag matrix. The size of the dots was about $3.4 \mathrm{~nm}$. Kern et al [3] showed that a submonolayer of oxygen on a $\mathrm{Cu}(110)$ surface could form stable periodic stripes of alternating oxygen overlayer and bare copper. The stripes had a width of about $10 \mathrm{~nm}$ and ran in the $\langle 001\rangle$ direction. Plass et al [4] found that a monolayer of $\mathrm{Cu}$ and $\mathrm{Pb}$ on a $\mathrm{Cu}(111)$ surface could form ordered patterns of dots or stripes, depending on the percentage of $\mathrm{Pb}$ atoms in the epilayer. Many ternary semiconductor epilayers demonstrated lateral composition modulation, such as (In, Ga)P on GaAs [5], AlInAs on InP [6] and GaAsN on GaAs [7]. The typical feature size is in the range of $10-50 \mathrm{~nm}$. Ordered patterns have been observed in other systems involving different mechanisms, such as electropolishing; see the work of Yuzhakov et al [8].

\footnotetext{
1 Author to whom any correspondence should be addressed.
}

The observations include nanoscopic phases and stable patterns. The phase sizes and the spatial ordering are determined by two competing actions: coarsening due to phase boundary energy and refining due to substrate-mediated elastic interaction. To illustrate the idea, consider a bulk two-phase alloy. When the alloy is annealed, atoms diffuse to reduce the surface area and the corresponding surface energy. The phases will coarsen until only one large particle is left in the matrix. However, the situation is different for a multi-phase epilayer on an elastic substrate, where the surface stress causes a phase refining action [9-11]. The nonuniform surface stress induces a fringe elastic field in the substrate, whose depth scales with the phase size. When the phase size is refined, the fringe field depth is reduced and so is the elastic energy. It is this reduction in the elastic energy that drives phase refining. This refining action competes with the coarsening process due to interfacial energy. As a result, the phases reach an equilibrium size. Moreover, the competition of two actions minimizes the total free energy, and causes the system to self-assemble into patterns.

We recently developed a phase field model to account for the nanoscale self-assembly behaviour of binary epilayers [1215]. Unlike Vanderbilt and co-workers [9, 10], we do not preassume the pattern types. Our model is a dynamic model, and the material system can generate whatever patterns it favours. We have studied a binary epilayer, 
where we performed a linear stability analysis [11] and numerical simulation [13-15]. Researchers have studied pattern formation in other systems. For instance, Chang's group determined when the stripe and dot patterns could form in an electropolishing process by a weak nonlinear analysis [8]. This paper extends our work to ternary epilayers. The third component may be used to effectively influence the growth of nanoscale patterns. A set of nonlinear diffusion equations couples the two concentration fields in the epilayer and the stress field in the substrate. Numerical simulations have revealed remarkably rich dynamics and suggest a significant degree of experimental control in growing ordered nanoscale structures.

\section{A model for the self-assembly of multi-phase epilayers}

This section develops a continuous phase field model for the self-assembly of a ternary epilayer on a solid substrate. Imagine an epilayer of three atomic species $\mathrm{A}, \mathrm{B}$, and $\mathrm{C}$ on a substrate of atomic species $\mathrm{S}$. The substrate is a semi-infinite elastic body occupying the half space below the $x_{1}-x_{2}$ plane $\left(x_{3}<0\right)$. The epilayer is treated as a surface object. We assume that the epilayer is a substitutional alloy of species $\mathrm{A}, \mathrm{B}$ and $\mathrm{C}$, and the atomic diffusion is confined within the epilayer.

\subsection{Kinematics}

Two sets of kinematic quantities describe the configuration of the epilayer-substrate composite: one for elastic deformation, and the other for mass transport. The epilayer-substrate as a thermodynamic system can vary by two means [12]: elastic displacement variation $\delta u_{i}$, and atomic relocation variation $\delta I_{\alpha}^{\mathrm{A}}$ and $\delta I_{\alpha}^{\mathrm{B}}$. In this paper a Latin letter in a subscript runs from 1 to 3 and a Greek letter in a subscript runs from 1 to 2 . The meaning of atomic relocation vector is briefly explained in the following. Imagine a curve in the surface of the epilayer. When some number of $\mathrm{A}$ atoms cross the curve, to maintain a flat epilayer, an equal number of $\mathrm{B}$ and $\mathrm{C}$ atoms must cross in the opposite direction. Let $m$ be the unit vector lying in the surface normal to the curve. The atomic relocation vector $\mathbf{I}^{\mathrm{A}}$ is defined such that $I_{\alpha}^{\mathrm{A}} m_{\alpha}^{\mathrm{A}}$ is the number of $\mathrm{A}$ atoms across a unit length of the curve. The vector $\mathbf{I}^{\mathrm{B}}$ is defined in a similar manner. We denote the atomic fraction of component $\mathrm{A}$ and $\mathrm{B}$ in the epilayer by $C_{1}$ and $C_{2}$, respectively. The fraction of atomic sites in the epilayer occupied by species $\mathrm{C}$ is $1-C_{1}-C_{2}$ since the epilayer is a substitutional alloy. We assume that the concentration fields $C_{1}\left(x_{1}, x_{2}, t\right)$ and $C_{2}\left(x_{1}, x_{2}, t\right)$ are time-dependent and spatially continuous. Due to mass conservation, the variation in the concentration fields relates to the variation in the atomic relocation by $\Lambda \delta C_{1}=-\delta I_{\alpha, \alpha}^{\mathrm{A}}$ and $\Lambda \delta C_{2}=-\delta I_{\alpha, \alpha}^{\mathrm{B}}$, where $\Lambda$ is the number of atoms per unit area on the surface. The quantity $\mathbf{I}^{\mathrm{A}}$ (or $\mathbf{I}^{\mathrm{B}}$ ) parallels a more familiar quantity, the atomic flux $\mathbf{J}^{\mathrm{A}}$ ( or $\mathbf{J}^{\mathrm{B}}$ ), in the same way as the displacement parallels the velocity. Due to mass conservation, the time rate of the concentration compensates the divergence of the flux vector, namely,

$$
\begin{aligned}
& \Lambda \partial C_{1} / \partial t=-J_{\alpha, \alpha}^{\mathrm{A}}, \\
& \Lambda \partial C_{2} / \partial t=-J_{\alpha, \alpha}^{\mathrm{B}} .
\end{aligned}
$$

\subsection{Energetics}

The free energy of the system consists of the surface energy plus the bulk elastic energy, namely,

$$
G=\int \Gamma \mathrm{d} A+\int W \mathrm{~d} V
$$

where $\Gamma$ is the surface energy per unit area of the epilayer, and $W$ the elastic energy per unit volume of the substrate. The area and volume are measured in the undeformed configuration of an infinite substrate. Generally speaking, the surface energy per unit area, $\Gamma$, is a function of the concentrations $C_{1}, C_{2}$, the concentration gradients $C_{1, \alpha}, C_{2, \alpha}$, and the strain in the surface, $\varepsilon_{\alpha \beta}$. The surface energy can be expanded by

$$
\Gamma=g+h_{1} C_{1, \alpha} C_{1, \alpha}+h_{2} C_{2, \alpha} C_{2, \alpha}+f \varepsilon_{\beta \beta},
$$

where $g, h_{1}, h_{2}$ and $f$ are functions of $C_{1}$ and $C_{2}$. Here we have assumed isotropy within the plane of the surface. The leading-order term in the concentration gradient is quadratic because, by symmetry, the term linear in the concentration gradient does not affect the surface energy.

When the concentration field is uniform in the epilayer, the substrate is unstrained, and the function $g\left(C_{1}, C_{2}\right)$ is the only remaining term. Thus it represents the surface energy per unit area of the uniform epilayer on the unstrained substrate. For a ternary material system, we adopt Muggianu's equation [16]:

$$
\begin{aligned}
& g\left(C_{1}, C_{2}\right)=C_{1} g_{\mathrm{A}}+C_{2} g_{\mathrm{B}}+\left(1-C_{1}-C_{2}\right) g_{\mathrm{C}}+\bar{g}\left(C_{1}, C_{2}\right), \\
& \bar{g}\left(C_{1}, C_{2}\right)=\Lambda k_{\mathrm{B}} T\left\{C_{1} \ln C_{1}+C_{2} \ln C_{2}\right. \\
& \quad+\left(1-C_{1}-C_{2}\right) \ln \left(1-C_{1}-C_{2}\right) \\
& \quad+C_{1} C_{2}\left[\Omega_{12}^{0}+\Omega_{12}^{1}\left(C_{1}-C_{2}\right)\right] \\
& \quad+C_{2}\left(1-C_{1}-C_{2}\right)\left[\Omega_{23}^{0}+\Omega_{23}^{1}\left(C_{1}+2 C_{2}-1\right)\right] \\
& \left.\quad+C_{1}\left(1-C_{1}-C_{2}\right)\left[\Omega_{13}^{0}+\Omega_{13}^{1}\left(2 C_{1}+C_{2}-1\right)\right]\right\},
\end{aligned}
$$

where $g_{\mathrm{A}}, g_{\mathrm{B}}$ and $g_{\mathrm{C}}$ are the excess energy when the epilayer is composed of pure $\mathrm{A}, \mathrm{B}$ or $\mathrm{C}$. The average concentration is constant due to mass conservation. Hence the linear term of excess energy does not affect diffusion and can be neglected. The remaining term $\bar{g}\left(C_{1}, C_{2}\right)$ represents the contribution from the entropy of mixing and the energy of mixing. In equation (4), $k_{\mathrm{B}}$ is Boltzmann's constant and $T$ is the absolute temperature. The dimensionless numbers $\Omega_{12}^{0}, \Omega_{12}^{1}, \Omega_{23}^{0}$, $\Omega_{23}^{1}, \Omega_{13}^{0}$ and $\Omega_{13}^{1}$, which measure bond strengths relative to the thermal energy $k_{\mathrm{B}} T$, control the shape of the function. Figure 1 shows the $3 \mathrm{D}$ surface of the function $\bar{g}\left(C_{1}, C_{2}\right)$ with parameters $\Omega_{12}^{0}=2.9, \Omega_{12}^{1}=0, \Omega_{23}^{0}=2.9, \Omega_{23}^{1}=0$, $\Omega_{13}^{0}=2.9$ and $\Omega_{13}^{1}=0$. The surface has three wells with this parameter combination. The function $\bar{g}\left(C_{1}, C_{2}\right)$ is symmetric about $C_{1}$ and $C_{2}$ with these parameters, and reaches minima at $\left(C_{1}, C_{2}\right)=(0.116,0.116),(0.768,0.116)$, and $(0.116,0.768)$. The contours of $\bar{g}\left(C_{1}, C_{2}\right)$ are shown in figure 2 for (a) $\Omega_{12}^{0}=2.9, \Omega_{12}^{1}=0, \Omega_{23}^{0}=2.9, \Omega_{23}^{1}=0$, $\Omega_{13}^{0}=2.9, \Omega_{13}^{1}=0$ and (b) $\Omega_{12}^{0}=2.9, \Omega_{12}^{1}=0.7, \Omega_{23}^{0}=2.9$, $\Omega_{23}^{1}=0.7, \Omega_{13}^{0}=2.9, \Omega_{13}^{1}=-0.7$. The contour is symmetric about a symmetry line with the parameters in (a), and is twisted with the non-zero $\Omega_{12}^{1}, \Omega_{23}^{1}, \Omega_{13}^{1}$ in (b).

In the phase field model, the second and third term in equation (3) represent the phase boundary energy, which drives phase coarsening. We assume that $h_{1}\left(C_{1}, C_{2}\right)$ and $h_{2}\left(C_{1}, C_{2}\right)$ 
Self-organized nanostructures in multi-phase epilayers

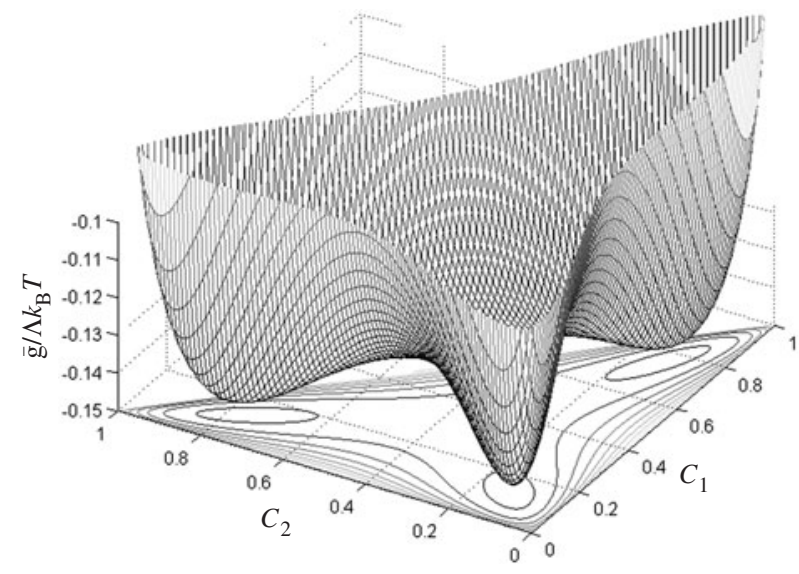

Figure 1. The 3D surface of the function $\bar{g}\left(C_{1}, C_{2}\right)$ with parameters $\Omega_{12}^{0}=2.9, \Omega_{12}^{1}=0, \Omega_{23}^{0}=2.9, \Omega_{23}^{1}=0, \Omega_{13}^{0}=2.9$ and $\Omega_{13}^{1}=0$.
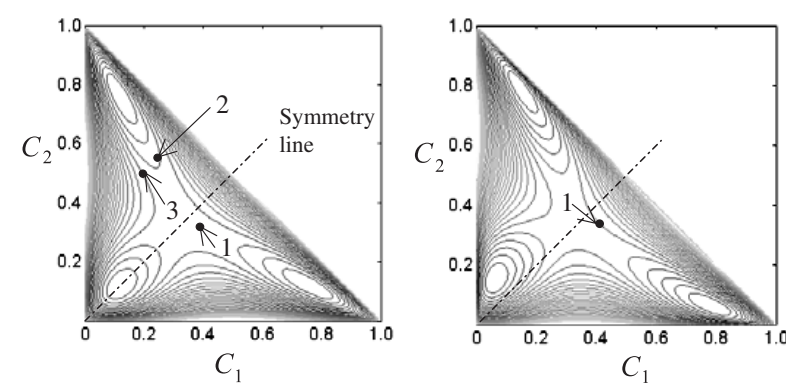

Figure 2. The contours of the function $\bar{g}\left(C_{1}, C_{2}\right)$ with parameters (a) $\Omega_{12}^{0}=2.9, \Omega_{12}^{1}=0, \Omega_{23}^{0}=2.9, \Omega_{23}^{1}=0, \Omega_{13}^{0}=2.9, \Omega_{13}^{1}=0$ and (b) $\Omega_{12}^{0}=2.9, \Omega_{12}^{1}=0.7, \Omega_{23}^{0}=2.9, \Omega_{23}^{1}=0.7, \Omega_{13}^{0}=2.9$, $\Omega_{13}^{1}=-0.7$. Positions 1,2 and 3 correspond to $\left(C_{1}, C_{2}\right)=(0.4,0.35),(0.25,0.55)$ and $(0.2,0.5)$, respectively.

are positive constants, i.e. $h_{1}\left(C_{1}, C_{2}\right)=h_{1}$ and $h_{2}\left(C_{1}, C_{2}\right)=$ $h_{2}$.

The change in the surface energy per unit strain, $f$, is known as surface stress $[17,18]$. It can be interpreted as the residual stress in the surface. More precisely, it is the resultant force per unit length. The surface stress is assumed to be a linear function of concentrations $C_{1}$ and $C_{2}$, namely [14],

$$
f\left(C_{1}, C_{2}\right)=\psi+\phi C_{1}+\eta C_{2},
$$

where $\psi$ is the surface stress when the epilayer comprises pure $\mathrm{C}$ atoms. $\phi$ and $\eta$ are the slopes. When the concentration fields are nonuniform in the epilayer, the surface stress is also nonuniform, which induces an elastic field in the substrate. This elastic field drives phase refining.

\subsection{Kinetics}

Finally we specify the kinetics, namely, the rate at which the configuration changes. The system evolves by making two kinds of changes: elastic deformation in the substrate, and atomic relocation in the epilayer. Elastic deformation does not dissipate energy, but mass transport does. Define the driving force $F_{\alpha}^{\mathrm{A}}$ (or $F_{\alpha}^{\mathrm{B}}$ ) as the reduction of the free energy of the system when an atom A (or B) relocates by unit distance. Following Cahn [19], we assume that the atomic flux is linearly proportional to the driving force:

$$
\begin{aligned}
& J_{\alpha}^{\mathrm{A}}=M_{1} F_{\alpha}^{\mathrm{A}}, \\
& J_{\alpha}^{\mathrm{B}}=M_{2} F_{\alpha}^{\mathrm{B}},
\end{aligned}
$$

where $M_{1}$ and $M_{2}$ are the mobilities of atoms in the epilayer.

\subsection{Variational statement and partial differential equations}

We now mix the ingredients. The energy variation of equation (2) gives

$$
\begin{aligned}
\delta G & =\int f \delta u_{\alpha, \alpha} \mathrm{d} A+\int \sigma_{i j} \delta u_{i, j} \mathrm{~d} V \\
& +\int \frac{\partial}{\Lambda \partial x_{\alpha}}\left(\frac{\partial \bar{g}}{\partial C_{1}}+\frac{\partial f}{\partial C_{1}} \varepsilon_{\beta \beta}-2 h_{1} \nabla^{2} C_{1}\right) \delta I_{\alpha}^{\mathrm{A}} \mathrm{d} A \\
& +\int \frac{\partial}{\Lambda \partial x_{\alpha}}\left(\frac{\partial \bar{g}}{\partial C_{2}}+\frac{\partial f}{\partial C_{2}} \varepsilon_{\beta \beta}-2 h_{2} \nabla^{2} C_{2}\right) \delta I_{\alpha}^{\mathrm{B}} \mathrm{d} A .
\end{aligned}
$$

Elastic deformation does not dissipate energy. The free energy variation with the elastic displacement vanishes, leading to

$$
\sigma_{i j, j}=0
$$

in the substrate and

$$
\sigma_{3 \alpha}=f_{, \alpha}, \quad \sigma_{33}=0
$$

on the surface. Equation (9) is the boundary condition for the elastic field in the substrate. The free energy variation with the atomic relocation defines the driving forces:

$$
\begin{aligned}
& F_{\alpha}^{\mathrm{A}}=-\frac{\partial}{\Lambda \partial x_{\alpha}}\left(\frac{\partial \bar{g}}{\partial C_{1}}+\frac{\partial f}{\partial C_{1}} \varepsilon_{\beta \beta}-2 h_{1} \nabla^{2} C_{1}\right), \\
& F_{\alpha}^{\mathrm{B}}=-\frac{\partial}{\Lambda \partial x_{\alpha}}\left(\frac{\partial \bar{g}}{\partial C_{2}}+\frac{\partial f}{\partial C_{2}} \varepsilon_{\beta \beta}-2 h_{2} \nabla^{2} C_{2}\right) .
\end{aligned}
$$

A combination of equations (1), (6) and (8)-(10) gives a set of nonlinear diffusion equations:

$$
\begin{aligned}
\frac{\partial C_{1}}{\partial t} & =\frac{M_{1}}{\Lambda^{2}} \nabla^{2}\left(\frac{\partial \bar{g}}{\partial C_{1}}+\frac{\partial f}{\partial C_{1}} \varepsilon_{\beta \beta}-2 h_{1} \nabla^{2} C_{1}\right), \\
\frac{\partial C_{2}}{\partial t} & =\frac{M_{2}}{\Lambda^{2}} \nabla^{2}\left(\frac{\partial \bar{g}}{\partial C_{2}}+\frac{\partial f}{\partial C_{2}} \varepsilon_{\beta \beta}-2 h_{2} \nabla^{2} C_{2}\right) .
\end{aligned}
$$

The non-uniform surface stress generates a traction field on the surface (see equation (9)), and this traction field induces an elastic field in the substrate. Cerruti [20] solved the elastic field in a half-space caused by a tangential point force acting on the surface. The elastic field generated by the distributed traction on the surface is given by a linear superposition:

$$
\begin{aligned}
\varepsilon_{\beta \beta} & =-\frac{\left(1-v^{2}\right) \phi}{\pi E} \iint \frac{\left(x_{1}-\xi_{1}\right) \frac{\partial C_{1}}{\partial \xi_{1}}+\left(x_{2}-\xi_{2}\right) \frac{\partial C_{1}}{\partial \xi_{2}}}{\left[\left(x_{1}-\xi_{1}\right)^{2}+\left(x_{2}-\xi_{2}\right)^{2}\right]^{3 / 2}} \mathrm{~d} \xi_{1} \mathrm{~d} \xi_{2} \\
& -\frac{\left(1-v^{2}\right) \eta}{\pi E} \iint \frac{\left(x_{1}-\xi_{1}\right) \frac{\partial C_{2}}{\partial \xi_{1}}+\left(x_{2}-\xi_{2}\right) \frac{\partial C_{2}}{\partial \xi_{2}}}{\left[\left(x_{1}-\xi_{1}\right)^{2}+\left(x_{2}-\xi_{2}\right)^{2}\right]^{3 / 2}} \mathrm{~d} \xi_{1} \mathrm{~d} \xi_{2},
\end{aligned}
$$

where $E$ is Young's modulus and $v$ Poisson's ratio of the substrate.

The diffusion equation (11) couples the two concentration fields and the elastic field. Equations (4), (11) and (12) define the evolution of the system. Given the initial concentration field $C_{1}\left(x_{1}, x_{2}, 0\right)$ and $C_{2}\left(x_{1}, x_{2}, 0\right)$, these equations determine the subsequent concentration fields $C_{1}\left(x_{1}, x_{2}, t\right)$ and $C_{2}\left(x_{1}, x_{2}, t\right)$. The following section details the simulation technique. 


\section{Numerical simulation}

The infinite integration in equation (12) makes it inefficient to solve equation (11) by a finite difference method in real space. A better method is to solve the equation by Fourier transform, which converts the integral-differential equation into a regular partial differential equation. The integration operation, as well as the differentiation over space, is removed, and the evolution equation can be dramatically simplified. Thanks to the exponential convergence of the Fourier spectral discretization, it requires a significantly smaller number of grid points to resolve the solution to within a prescribed accuracy [21].

Now we normalize the length and time of the system. A length scale is defined by a comparison of the first and the last term in the parentheses in equation $(11 a)$, which is

$$
b=\left(\frac{h_{1}}{\Lambda k_{\mathrm{B}} T}\right)^{1 / 2} .
$$

In the Cahn-Hilliard model [19], this length scales the width of the phase boundary that the concentration changes from the level of one phase to that of the other. The magnitude of $h_{1}$ is of the order of energy per atom at a phase boundary. Taking the magnitude of $h_{1} \sim 1 \mathrm{eV}, \Lambda \sim 5 \times 10^{19} \mathrm{~m}^{-2}$, and $k_{\mathrm{B}} T \sim 5 \times 10^{-21} \mathrm{~J}$ (corresponding to $T=400 \mathrm{~K}$ ), we have $b \sim 0.6 \mathrm{~nm}$. Other lengths are defined by comparing the second and the last terms in the parentheses in equation (11), namely

$$
\begin{gathered}
l_{1}=\frac{E h_{1}}{\left(1-v^{2}\right) \phi^{2}}, \quad l_{2}=\frac{E h_{1}}{\left(1-v^{2}\right) \phi \eta}, \\
l_{3}=\frac{E h_{1}}{\left(1-v^{2}\right) \eta^{2}} .
\end{gathered}
$$

Young's modulus of a bulk solid is about $E \sim 10^{11} \mathrm{~N} \mathrm{~m}^{-2}$. According to Ibach [22], the slope of the surface stress is of the order $\phi \sim 4 \mathrm{~N} \mathrm{~m}^{-1}$. These magnitudes, together with $h_{1} \sim 1 \mathrm{eV}$, give $l_{1} \sim 0.6 \mathrm{~nm}$. The lengths $l_{2}$ and $l_{3}$ are of the same order.

From equation (11a), disregarding a dimensionless factor, we note that the diffusivity scales as $D \sim M_{1} k_{\mathrm{B}} T / \Lambda$. To resolve the event occurring over the length scale $b$, the time scale is $\tau=b^{2} / D$, namely,

$$
\tau=\frac{h_{1}}{M_{1}\left(k_{\mathrm{B}} T\right)^{2}} .
$$

Normalize the coordinates by $b$ and the time by $\tau$. Equations (4), (11) and (12) combine into a set of normalized diffusion equations. Denote the Fourier transform of $C_{1}\left(x_{1}, x_{2}, t\right)$ by $\hat{C}_{1}\left(k_{1}, k_{2}, t\right)$, where $\left(k_{1}, k_{2}\right)$ are the coordinates in Fourier space. Then

$$
\hat{C}_{1}\left(k_{1}, k_{2}, t\right)=\int_{-\infty}^{\infty} \int_{-\infty}^{\infty} C_{1}\left(x_{1}, x_{2}, t\right) \mathrm{e}^{-\mathrm{i}\left(k_{1} x_{1}+k_{2} x_{2}\right)} \mathrm{d} x_{1} \mathrm{~d} x_{2} .
$$

Taking the Fourier transform on the normalized diffusion equations gives

$$
\begin{gathered}
\frac{\partial \hat{C}_{1}}{\partial t}=-k^{2} \hat{P}_{1}-2 k^{4} \hat{C}_{1}+2 k^{3} Q_{1} \hat{C}_{1}+2 k^{3} Q_{2} \hat{C}_{2}, \\
\frac{\partial \hat{C}_{2}}{\partial t}=S\left(-k^{2} \hat{P}_{2}-2 k^{4} H \hat{C}_{2}+2 k^{3} Q_{2} \hat{C}_{1}+2 k^{3} Q_{3} \hat{C}_{2}\right),
\end{gathered}
$$

where $k=\sqrt{k_{1}^{2}+k_{2}^{2}}, Q_{1}=b / l_{1}, Q_{2}=b / l_{2}, Q_{3}=b / l_{3}$, $S=M_{2} / M_{1}$ and $H=h_{2} / h_{1} . \quad \hat{C}_{2}\left(k_{1}, k_{2}, t\right), \hat{P}_{1}\left(k_{1}, k_{2}, t\right)$ and $\hat{P}_{2}\left(k_{1}, k_{2}, t\right)$ denote the Fourier transform of $C_{2}\left(x_{1}, x_{2}, t\right)$, $P_{1}\left(x_{1}, x_{2}, t\right)$ and $P_{2}\left(x_{1}, x_{2}, t\right)$, respectively. Details about the derivation of strain fields in Fourier space can be found in [15]. The function $P_{1}\left(C_{1}, C_{2}\right)$ comes from the derivative of $\bar{g}\left(C_{1}, C_{2}\right) / \Lambda k_{\mathrm{B}} T$ with respect to $C_{1}$, and $P_{2}\left(C_{1}, C_{2}\right)$ comes from the derivative of $\bar{g}\left(C_{1}, C_{2}\right) / \Lambda k_{\mathrm{B}} T$ with respect to $C_{2}$. The expressions are

$$
\begin{aligned}
& P_{1}(\left.C_{1}, C_{2}\right)=\ln \left(\frac{C_{1}}{1-C_{1}-C_{2}}\right) \\
&+C_{2}\left\{\Omega_{12}^{0}+\Omega_{12}^{1}\left(2 C_{1}-C_{2}\right)\right\} \\
&-C_{2}\left\{\Omega_{23}^{0}+\Omega_{23}^{1}\left(2 C_{1}+3 C_{2}-2\right)\right\} \\
&+\Omega_{13}^{0}\left(1-2 C_{1}-C_{2}\right) \\
&+\Omega_{13}^{1}\left(6 C_{1}+2 C_{2}-6 C_{1}^{2}-C_{2}^{2}-6 C_{1} C_{2}-1\right), \\
& P_{2}\left(C_{1}, C_{2}\right)=\ln \left(\frac{C_{2}}{1-C_{1}-C_{2}}\right) \\
&+C_{1}\left\{\Omega_{12}^{0}+\Omega_{12}^{1}\left(C_{1}-2 C_{2}\right)\right\} \\
&-C_{1}\left\{\Omega_{13}^{0}+\Omega_{13}^{1}\left(3 C_{1}+2 C_{2}-2\right)\right\} \\
&+\Omega_{23}^{0}\left(1-C_{1}-2 C_{2}\right) \\
&+\Omega_{23}^{1}\left(2 C_{1}+6 C_{2}-C_{1}^{2}-6 C_{2}^{2}-6 C_{1} C_{2}-1\right) .
\end{aligned}
$$

The next consideration is the time variable in equation (17) Instead of the explicit forward Euler method, which requires a very small time step to maintain stability, we employ a semi-implicit scheme proposed by Chen and Shen [21]. We treat the linear term implicitly to reduce the stability constraint, while we still treat the nonlinear term explicitly to avoid solving nonlinear equations at each time step. For a given time $t$ and a time step $\Delta t$, we denote $\hat{C}_{1}^{n}=\hat{C}_{1}\left(k_{1}, k_{2}, t\right), \hat{C}_{1}^{n+1}=\hat{C}_{1}\left(k_{1}, k_{2}, t+\Delta t\right), \hat{P}_{1}^{n}=$ $\hat{P}_{1}\left(k_{1}, k_{2}, t\right), \hat{C}_{2}^{n}=\hat{C}_{2}\left(k_{1}, k_{2}, t\right), \hat{C}_{2}^{n+1}=\hat{C}_{2}\left(k_{1}, k_{2}, t+\Delta t\right)$, and $\hat{P}_{2}^{n}=\hat{P}_{2}\left(k_{1}, k_{2}, t\right)$. In equation (17), we replace $\hat{C}_{1}$ by $\hat{C}_{1}^{n+1}, \partial \hat{C}_{1} / \partial t$ by $\left(\hat{C}_{1}^{n+1}-\hat{C}_{1}^{n}\right) / \Delta t$, and $\hat{P}_{1}$ by $\hat{P}_{1}^{n}$, and treat $\hat{C}_{2}$, $\partial \hat{C}_{2} / \partial t, \hat{P}_{2}$ in the same way. Then, we obtain the simulation algorithm in a discretized matrix form:

$$
\begin{aligned}
& \left\{\begin{array}{c}
\hat{C}_{1}^{n+1} \\
\hat{C}_{2}^{n+1}
\end{array}\right\} \\
& =\left[\begin{array}{cc}
1+\left(2 k^{4}-2 k^{3} Q_{1}\right) \Delta t & -2 \Delta t k^{3} Q_{2} \\
-2 \Delta t S k^{3} Q_{2} & 1+S\left(2 H k^{4}-2 k^{3} Q_{3}\right) \Delta t
\end{array}\right]^{-1} \\
& \left.\quad \times\left[\begin{array}{c}
\hat{C}_{1}^{n} \\
\hat{C}_{2}^{n}
\end{array}\right\}-k^{2} \Delta t\left\{\begin{array}{c}
\hat{P}_{1}^{n} \\
S \hat{P}_{2}^{n}
\end{array}\right\}\right] .
\end{aligned}
$$

For a simulation with a given time step, the coefficient matrix on the right-hand side of equation (19) is constant. Hence it is only necessary to perform the matrix inverse operation once, which is a significant benefit from the semi-implicit scheme.

The simulation is carried out in a square cell of size $L \times L$ in real space $\left(x_{1}, x_{2}\right)$. The periodic boundary condition is applied to replicate the cell to the entire surface. The cell size must be large enough to contain sufficient numbers of features but small enough to shorten the computation time. According to the linear perturbation analysis [11], the equilibrium wavelength 
is estimated as $4 \pi l_{1}$. We choose a cell size of the order $L \sim 200 l_{1}$ in our simulation. The cell is divided into $N \times N$ grids. The grid space $\Delta=L / N$ should be small enough to describe the phase boundary. We choose $\Delta=b$ in our simulation. The corresponding cell size in Fourier space $\left(k_{1}, k_{2}\right)$ is $2 \pi / \Delta \times 2 \pi / \Delta$. The corresponding grid space is $2 \pi / L$. To connect the values of $C_{1}, C_{2}, P_{1}$ and $P_{2}$ at the grid points in real space to those in Fourier space, we adopt the fast Fourier transform (FFT). FFT exploits various properties of the Fourier transform to enable the transformation to be done in $\mathrm{O}\left(N \log _{2} N\right)$ operations instead of $\mathrm{O}\left(N^{2}\right)$ operations. The input contains the initial concentration distribution and the parameters $Q_{1}, Q_{2}, Q_{3}, S, H, \Omega_{12}^{0}, \Omega_{12}^{1}, \Omega_{23}^{0}, \Omega_{23}^{1}, \Omega_{13}^{0}$, $\Omega_{13}^{1}$. At each time step, the values of $P_{1}^{n}, P_{2}^{n}$ at each grid point are calculated with equation (4). Then $C_{1}^{n}, C_{2}^{n}$ and $P_{1}^{n}, P_{2}^{n}$ are transformed to $\hat{C}_{1}^{n}, \hat{C}_{2}^{n}$ and $\hat{P}_{1}^{n}, \hat{P}_{2}^{n}$ by FFT. $\hat{C}_{1}^{n+1}$ and $\hat{C}_{2}^{n+1}$ are updated by equation (19). With the inverse FFT, $C_{1}^{n+1}$ and $C_{2}^{n+1}$ in real space are obtained. The simulation advances by repeating the procedure.

\section{Results and discussions}

The free energy $\bar{g}\left(C_{1}, C_{2}\right)$ induces phase separation. Its shape is controlled by several dimensionless parameters. We apply two sets of parameters in the simulation:

$$
\begin{aligned}
\text { (a) } \Omega_{12}^{0} & =2.9, \Omega_{12}^{1}=0, \Omega_{23}^{0}=2.9, \Omega_{23}^{1}=0, \Omega_{13}^{0}=2.9, \\
& \Omega_{13}^{1}=0 \text { and } \\
\text { (b) } \Omega_{12}^{0} & =2.9, \Omega_{12}^{1}=0.7, \Omega_{23}^{0}=2.9, \Omega_{23}^{1}=0.7, \Omega_{13}^{0}=2.9, \\
& \Omega_{13}^{1}=-0.7 .
\end{aligned}
$$

The free energy $\bar{g}\left(C_{1}, C_{2}\right)$ has three wells with these parameters. The bottoms of the three wells are located at $\left(C_{1}, C_{2}\right)=(0.116,0.116),(0.768,0.116),(0.116,0.768)$ for the first case and $\left(C_{1}, C_{2}\right)=(0.061,0.150),(0.789,0.061)$, $(0.150,0.789)$ for the latter. The contours are shown in figure 2 .

Figures 3-6 show selected simulation results. We choose $H=1$ (i.e. $h_{1}=h_{2}$ ) and $S=1$ (i.e. $M_{1}=M_{2}$ ) in the simulation. Other parameters are specified individually for each simulation. Each calculation cell contains $256 \times 256$ grids with grid size equal to $b$. Time is normalized by the time scale $\tau$. The time step used in the computation is $\Delta t=1.0 \times 10^{-2}$. At a given time the concentration fields in the $\left(x_{1}, x_{2}\right)$ plane are visualized by greyscale graphs. The darker region corresponds to higher concentration and the brighter region corresponds to lower concentration. The concentration fields $C_{1}\left(x_{1}, x_{2}\right)$ and $C_{2}\left(x_{1}, x_{2}\right)$ evolve over time, but the average concentrations over the calculation cell do not change thanks to mass conservation. We use $C_{1}^{\text {ave }}$ and $C_{2}^{\text {ave }}$ to represent the average concentration of $C_{1}$ and $C_{2}$, respectively. The average concentrations enforce a constraint to a self-assembly process. To examine the effect, the simulations in this paper involve $\left(C_{1}^{\text {ave }}, C_{2}^{\text {ave }}\right)=(0.4,0.35),(0.25,0.55)$ and $(0.2$, 0.5 ), as illustrated in figure 2 . The initial conditions are given by fluctuating randomly within 0.001 from the average concentrations.

Figure 3 shows an evolution sequence from $t=0$ to 1000 . The parameters $Q_{1}, Q_{2}, Q_{3}$ are taken to be 1, corresponding to a symmetric material system. $\Omega_{12}^{0}=2.9, \Omega_{12}^{1}=0, \Omega_{23}^{0}=2.9$, $\Omega_{23}^{1}=0, \Omega_{13}^{0}=2.9$ and $\Omega_{13}^{1}=0$ are chosen for the function $\bar{g}\left(C_{1}, C_{2}\right)$. The left-hand column plots the concentration field

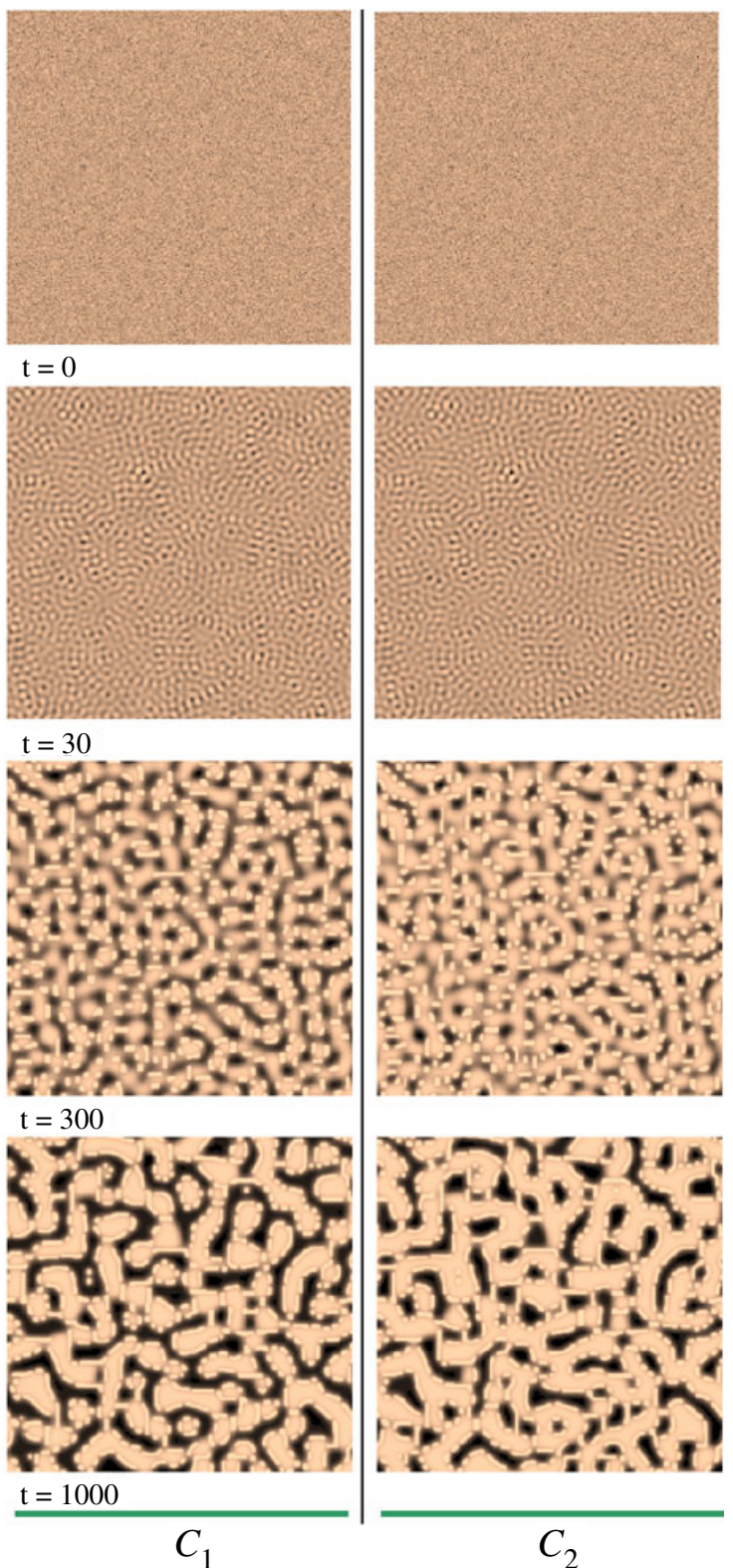

Figure 3. An evolution sequence from a random initial condition The average concentrations of $C_{1}$ and $C_{2}$ are 0.4 and 0.35 , respectively. The parameters $Q_{1}, Q_{2}, Q_{3}$ are taken to be 1 . $\Omega_{12}^{0}=2.9, \Omega_{12}^{1}=0, \Omega_{23}^{0}=2.9, \Omega_{23}^{1}=0, \Omega_{13}^{0}=2.9$ and $\Omega_{13}^{1}=0$

$C_{1}$ and the right-hand column plots the concentration field $C_{2}$. The simulation starts from a random initial condition with average concentrations of $C_{1}^{\text {ave }}=0.4$ and $C_{2}^{\text {ave }}=0.35$.

Shortly after the simulation starts, the amplitudes of the concentration fields rapidly evolve close to the equilibrium values of the three phases, i.e. $(0.116,0.116),(0.768,0.116)$, $(0.116,0.768)$. These equilibrium values are estimated by the $\bar{g}\left(C_{1}, C_{2}\right)$ term only. The competition of phase boundary energy and elastic energy mainly determines the phase sizes and their spatial ordering, having only minor influence on the equilibrium compositions. At $t=300$, we can clearly identify three phases by their shades. The dark and grey phases organize into a serpentine structure with a bright dot phase scattering in between. The $C_{2}$ graph on the right 

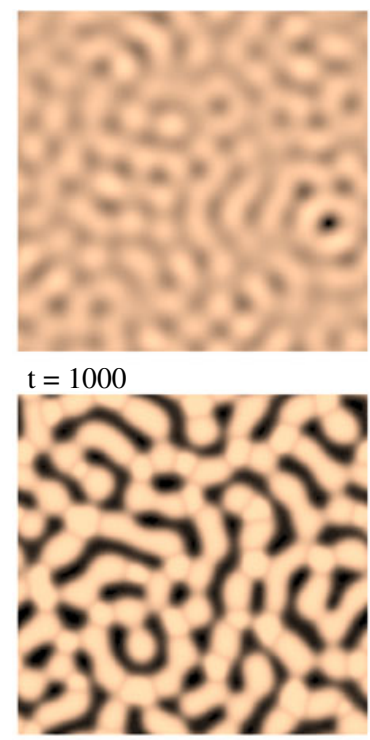

$\mathrm{t}=3000$

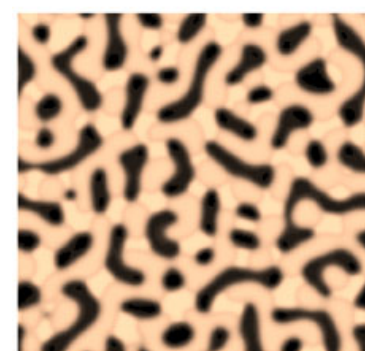

$\mathrm{t}=1000$

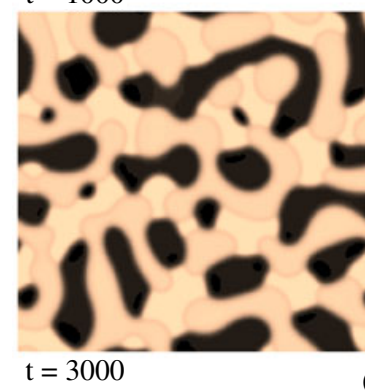

$C_{1}$
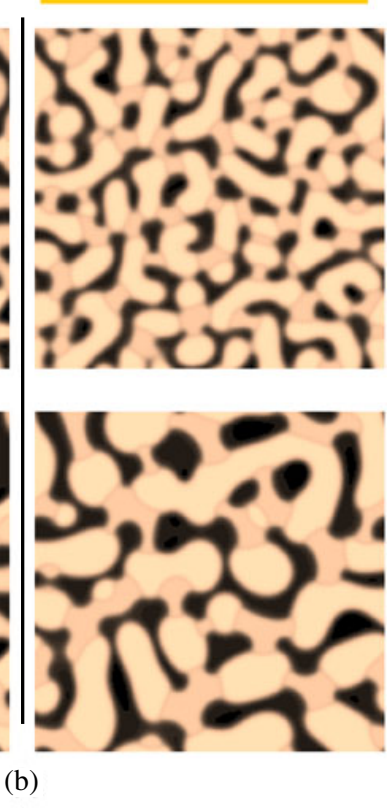

(b)

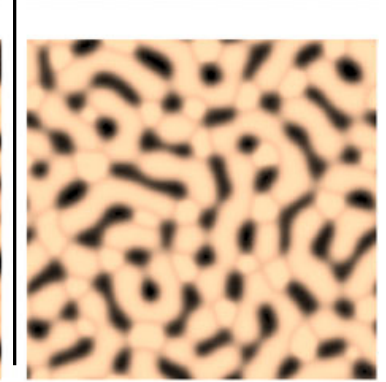

(a)

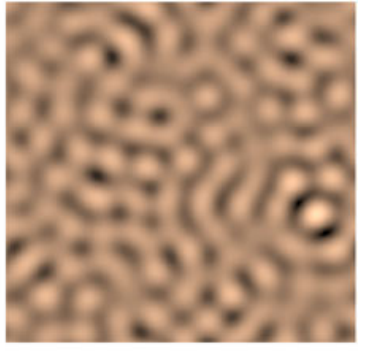

$C_{2}$

Figure 4. Two evolution sequences without surface stress $\left(Q_{1}=0\right.$, $Q_{2}=0, Q_{3}=0$ ). The average concentrations of $C_{1}$ and $C_{2}$ are 0.4 and 0.35 , respectively. The simulations start from the same initial conditions as those in figure 3. Other parameters are (a) $\Omega_{12}^{0}=2.9$, $\Omega_{12}^{1}=0, \Omega_{23}^{0}=2.9, \Omega_{23}^{1}=0, \Omega_{13}^{0}=2.9, \Omega_{13}^{1}=0$ and (b) $\Omega_{12}^{0}=2.9, \Omega_{12}^{1}=0.7, \Omega_{23}^{0}=2.9, \Omega_{23}^{1}=0.7, \Omega_{13}^{0}=2.9$, $\Omega_{13}^{1}=-0.7$.

is almost a negative of the $C_{1}$ graph on the left except for the bright dot phase. This suggests that the dark and grey phases have compositions complementary to each other, while the bright dot phase has a composition symmetric about $C_{1}$ and $C_{2}$. Thus the composition of the three phases can be identified. In the $C_{1}$ graph, the dark, grey and bright phases have compositions close to $\left(C_{1}, C_{2}\right)=(0.768,0.116),(0.116$, $0.768)$ and $(0.116,0.116)$, respectively. We can distinguish the grey and bright phases in the $C_{1}$ graph because the existence of elastic energy and phase boundary energy has shifted the
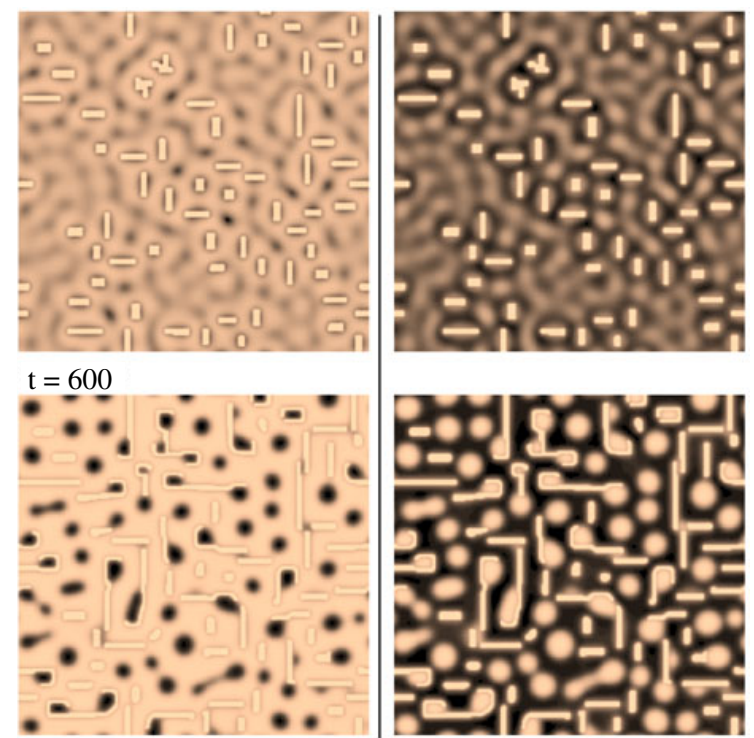

$\mathrm{t}=1000$

(a)

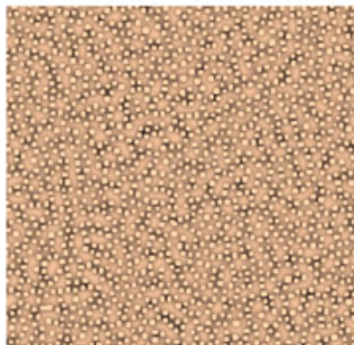

$\mathrm{t}=100$

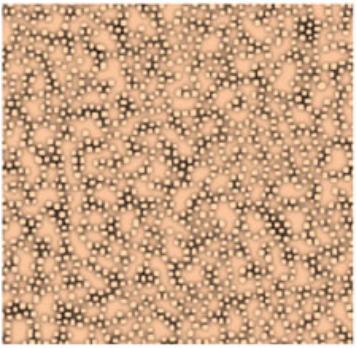

$\mathrm{t}=1000$

(b)
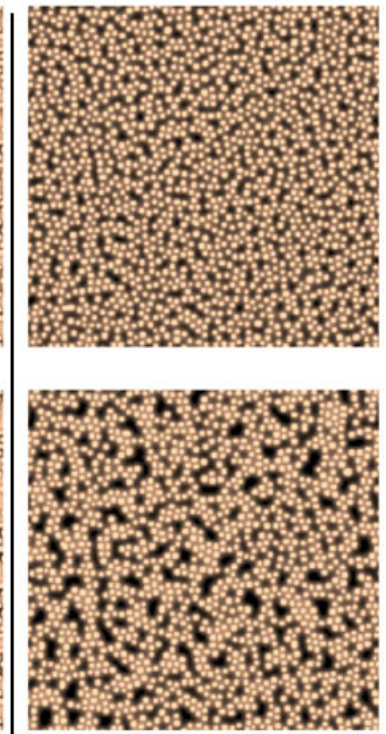

(b)

\section{$C_{1}$}

$C_{2}$

Figure 5. Two evolution sequences with random initial conditions. The parameters $Q_{1}, Q_{2}, Q_{3}$ are taken to be 1 . Other parameters are $\Omega_{12}^{0}=2.9, \Omega_{12}^{1}=0, \Omega_{23}^{0}=2.9, \Omega_{23}^{1}=0, \Omega_{13}^{0}=2.9$ and $\Omega_{13}^{1}=0$. The average concentrations are (a) $\left(C_{1}^{\text {ave }}, C_{2}^{\text {ave }}\right)=(0.25,0.55)$ and (b) $\left(C_{1}^{\text {ave }}, C_{2}^{\text {ave }}\right)=(0.2,0.5)$.

equilibrium compositions slightly from $C_{1}=0.116$ (estimated from $\bar{g}\left(C_{1}, C_{2}\right)$ only) to two different values. The dark, grey and bright phases in the $C_{2}$ graph have compositions close to $(0.116,0.768),(0.768,0.116)$, and $(0.116,0.116)$. Compared with the other two phases, the bright phase has quite small phase size (or stripe width). This can be explained in terms of the constraint of average concentration. Consider the concentration component $C_{3}$ of the three phases, namely $1-C_{1}-C_{2}$. The dark, grey and bright phases have $C_{3}=0.116$, 0.116 , and 0.768 , respectively. The average concentration $(0.4$, 0.35 ), or $C_{3}=0.25$, is closer to the composition of the dark and grey phases. As a result of mass conservation, the size 


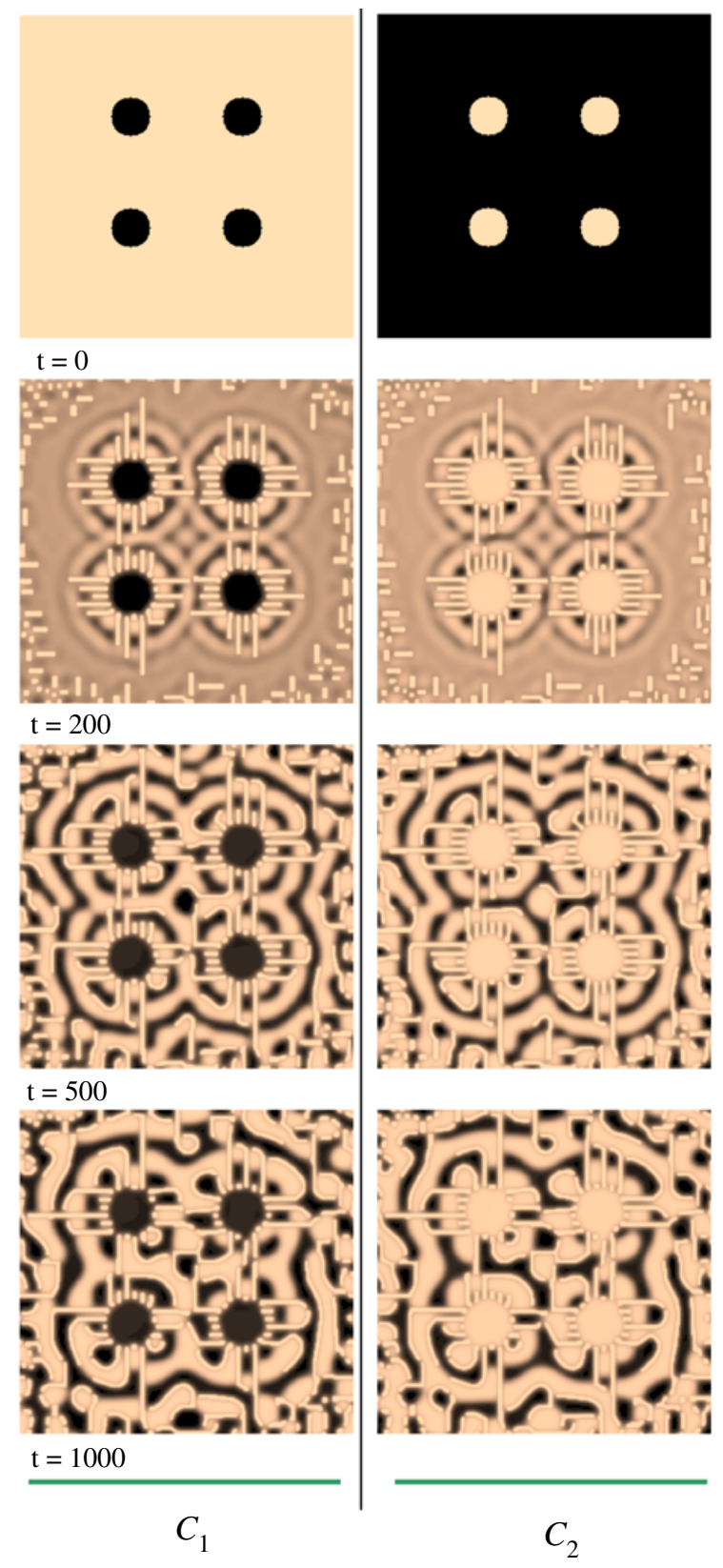

Figure 6. An evolution sequence with four guide discs. Initially, the background has average concentrations $C_{1}^{\text {ave }}=0.4$ and $C_{2}^{\text {ave }}=0.35$. The four guide discs are assigned the equilibrium concentrations $C_{1}=0.768$ and $C_{2}=0.116$. The parameters $Q_{1}, Q_{2}, Q_{3}$ are taken to be 1. Other parameters are $\Omega_{12}^{0}=2.9, \Omega_{12}^{1}=0, \Omega_{23}^{0}=2.9$, $\Omega_{23}^{1}=0, \Omega_{13}^{0}=2.9$ and $\Omega_{13}^{1}=0$.

of the bright phase has to be smaller. At time $t=1000$, the bright dots aggregate at the boundary of the dark and grey phases, forming narrow stripes separating the two phases. The serpentine structures have been observed experimentally in many bulk or film systems. The systems are typically isotropic in the plane of the film.

Figure 4 shows two evolution sequences without surface stress, i.e. $Q_{1}=0, Q_{2}=0, Q_{3}=0$. The simulations start from the same initial conditions as those in figure 3 with $\left(C_{1}^{\text {ave }}, C_{2}^{\text {ave }}\right)=(0.4,0.35)$. The two sequences in figure 4 use different parameters for $\bar{g}\left(C_{1}, C_{2}\right)$. Figure 4 (a) has $\Omega_{12}^{0}=2.9$,
$\Omega_{12}^{1}=0, \Omega_{23}^{0}=2.9, \Omega_{23}^{1}=0, \Omega_{13}^{0}=2.9, \Omega_{13}^{1}=0$, while figure $4(\mathrm{~b})$ has $\Omega_{12}^{0}=2.9, \Omega_{12}^{1}=0.7, \Omega_{23}^{0}=2.9, \Omega_{23}^{1}=0.7$, $\Omega_{13}^{0}=2.9$ and $\Omega_{13}^{1}=-0.7$. In both cases, the phases try to increase their sizes as much as possible, being restricted only by mass conservation and the size of calculation cell.

Now observe the $C_{1}$ and $C_{2}$ graphs at $t=1000$ in figure 4(a). The phases have compositions very close to the average concentration $(0.4,0.35)$, suggesting that phase separation is far from completion at this time. As a result, the phases look fuzzy and are barely distinguishable. In contrast, the phases attain their equilibrium compositions in less than $t=300$ in the sequence of figure 3, indicating that the presence of surface stress accelerates the evolution. The patterns in figure 3 also exhibit different features from those in figure 4(a), especially the formation and aggregation of dots. Figure 4(b) has larger phase sizes compared with figure 4(a), which suggests that the parameters in $\bar{g}\left(C_{1}, C_{2}\right)$ may influence the coarsening process. Such an influence is effected through the driving force.

Figure 5 shows two evolution sequences with different average concentrations. The simulations start from random initial conditions. Figure 5(a) has an average concentration $\left(C_{1}^{\text {ave }}, C_{2}^{\text {ave }}\right)=(0.25,0.55)$ and figure $5(\mathrm{~b})$ has $\left(C_{1}^{\text {ave }}, C_{2}^{\text {ave }}\right)=$ $(0.2,0.5) . Q_{1}, Q_{2}, Q_{3}$ are taken to be 1 in the simulation. Other parameters are $\Omega_{12}^{0}=2.9, \Omega_{12}^{1}=0, \Omega_{23}^{0}=2.9$, $\Omega_{23}^{1}=0, \Omega_{13}^{0}=2.9$ and $\Omega_{13}^{1}=0$. Narrow stripes and scattered dots are observed in figure 5 (a). In the $C_{1}$ graph, the bright stripes have composition close to $(0.116,0.116)$ and the dark dots have composition close to $(0.768,0.116)$. The grey phase has composition $(0.116,0.768)$. As is shown in figure 2 , the composition $(0.116,0.768)$ is the closest to the average concentration (see point 2 in figure 2) among the three. Hence the grey phase occupies most of the area. In the $C_{2}$ graph, the bright, grey and dark phases have compositions close to $(0.116$, $0.116),(0.768,0.116),(0.116,0.768)$, respectively. From $t=$ 600 to 1000 , the bright stripes extend their lengths and form a network-like structure. Figure 5(b) shows quite different patterns. The bright phase forms small densely packed dots instead of narrow stripes. A comparison of figures 3, 5(a) and (b) clearly demonstrates the important effect of average concentration on pattern formation.

Figure 6 shows an evolution sequence with four guide discs. The background has average concentrations $C_{1}^{\text {ave }}=0.4$ and $C_{2}^{\text {ave }}=0.35$. The guide discs are assigned the equilibrium concentrations $C_{1}=0.768$ and $C_{2}=0.116$. Consequently, they evolve little and serve as guidance to direct the selfassembly process. Interesting patterns are obtained. The example shows that the pattern at a finite time is influenced by the initial conditions. When a pattern at a coarse scale is introduced, for example by photolithography, the coarse pattern acts like a framework, which influences the selfassemblies on a fine scale. Diverse patterns may be produced this way.

\section{Concluding remarks}

This paper develops a continuous phase field model to account for the self-assembly behaviour of a ternary epilayer. The main ingredients for ordering a stable, ordered phase pattern include phase separation, phase coarsening and phase refining. These actions are incorporated into our model: 
(1) a three-well free energy drives phase separation;

(2) the phase boundary energy drives phase coarsening.; and

(3) the concentration-dependent surface stress drives phase refining.

A set of nonlinear diffusion equations is obtained, which couple the two concentration fields in the epilayer and the elastic field in the substrate. These equations are solved numerically by applying the fast Fourier transform and a semiimplicit method. Numerical simulations reveal remarkably rich dynamics in the self-assembly of ternary epilayers and suggest a significant degree of experimental control in the growth of nanoscale superlattices.

\section{Acknowledgment}

The authors acknowledge the financial support of a Faculty Research Grant from the University of Michigan.

\section{References}

[1] Binnig G and Rohrer H 2000 IBM J. Res. Dev. 44279

[2] Pohl K, Bartelt M C, de la Figuera J, Bartelt N C, Hrbek J and Hwang R Q 1999 Nature 397238
[3] Kern K, Niebus H, Schatz A, Zeppenfeld P, George J and Comsa G 1991 Phys. Rev. Lett. 67855

[4] Plass R, Last J A, Bartelt N C and Kellogg G L 2001 Nature 412875

[5] Follstaedt D M, Schneider R P and Jones E D 1994 J. Appl. Phys. 773077

[6] Francoeur S, Hanna M C, Norman A G and Mascarenhas A 2002 Appl. Phys. Lett. 80243

[7] Suemune I, Morooka N, Uesugi K, Ok Y-W and Seong T-Y 2000 J. Cryst. Growth 221546

[8] Yuzhakov V V, Chang H-C and Miller A E 1997 Phys. Rev. B 5612608

[9] Alerhand O L, Vanderbilt D, Meade R D and Joannopoulos J D 1988 Phys. Rev. Lett. 611973

[10] Ng K-O and Vanderbilt D 1995 Phys. Rev. B 522177

[11] Lu W and Suo Z 1999 Z. Metallk. 90956

[12] Suo Z and Lu W 2000 J. Mech. Phys. Solids 48211

[13] Lu W and Suo Z 2001 J. Mech. Phys. Solids 491937

[14] Lu W and Suo Z 2002 Phys. Rev. B 65085401

[15] Lu W and Suo Z 2002 Phys. Rev. B 65205418

[16] Saunders N and Miodounik N P 1998 CALPHAD-Calculation of Phase Diagrams (New York: Pergamon)

[17] Cahn J W 1980 Acta Metall. 281333

[18] Rice J R and Chuang T-J 1981 J. Am. Ceram. Soc. 6446

[19] Cahn J W and Hilliard J E 1958 J. Chem. Phys. 28258

[20] Johnson K L 1985 Contact Mechanics (Cambridge: Cambridge University Press)

[21] Chen L-Q and Shen J 1998 Comput. Phys. Commun. 108147

[22] Ibach H 1997 Surf. Sci. Rep. 29193 